\title{
Considerations in the Quality Control towards Translation of Extracellular Vesicles Derived from Eukaryotic Prokaryotic Cells
}

\author{
Haixia Wang ${ }^{1}$ Yanhong Gao ${ }^{2}$ and Jin Gao ${ }^{1 *}$ \\ ${ }^{1}$ Department of Pharmaceutical Sciences, Washington State University, USA \\ ${ }^{2}$ Shangqiu Municipal First Senior High School, China
}

*Corresponding author: Jin Gao, Department of Pharmaceutical Sciences, College of Pharmacy and Pharmaceutical Sciences, Washington State University, Spokane, USA.
Received Date: October 26, 2019

Published Date: November 04, 2019

\section{Introduction}

According to concept of ISEV (The international Society for Extracellular Vesicles), "extracellular vesicles (EVs) are defined as the particles naturally released from cells that are comprised of a lipid bilayer membrane [1]. Acting as important mediators between cells that regulate both physiological and pathological conditions in the living bodies, EVs are nanosized spherical compartments and contain lipids, proteins and various nucleic acids of their source cells $[2,3]$. Based on their biogenesis and sizes, EVs are generally categorized into three types, including exosomes, microvesicles and apoptotic bodies. In general, sizes of EVs vary within the range 30$5000 \mathrm{~nm}[4,5]$. Besides EVs derived from eukaryotes, prokaryotes also secrete EVs. It has been reported that Gram-positive and Gramnegative bacteria can both generate EVs [6-8]. The size of the outer membrane-derived vesicles (OMVs) from both Gram-positive and Gram-negative bacteria was reported to be around $20-100 \mathrm{~nm}$ in diameter [8,9]. Inspired by the generation of naturally secreted EVs, scientists are also seeking to prepare biomimetic EVs by physical $[10,11]$, and chemical $[12]$ methods in recent years. In general, the artificial EVs exhibit similar properties to the natural ones. As a supplementary to the natural EVs, biomimetic EVs possess some advantages in some aspects, such as purity, yield, targeting ability, etc. Either EVs or OMVs can perform as vaccines without loading. They also can be loaded by bioactive ingredients as drug delivery carriers. In the past decades, a lot of efforts had been made to translate EVs for clinical use. However, there are many challenges existing at each stage of commercialization of EVs. One of them is quality control. Generally, to control the quality of EVs as drug carriers or vaccines, the following aspects should be taken into consideration.
- Impurities. Safety is always the priority when quality is taken into consideration. Deriving from parent cells, EVs will unavoidably inherit some ingredients of their source cells. Obviously, some impurities, such as DNA [13] and RNA [14], are bioactive and may impair the functions of cargoes. Other potential impairments also need keeping an eye on include enzymes, kinases and some potential carcinoma-inducing agents especially when prepared from cancer source cells. Except endogenous impurities, the exogenous pathogens also need to be monitored when prepared from prokaryotic cells.

- Cargo content. When cargos, either small molecules, such as chemicals, or large molecules, such as proteins and RNAs, were loaded into EVs, they have to be assessed to guarantee the necessary bioactive ingredient levels. As regard to the cargo loading methods, there are some reviews can be referred $[9,15]$.

- $\quad$ Targeting ability. As drug carrier, the content of targeting ligands [15] on the EVs should be evaluated. The targeting capability of EVs also need assessment both in vitro and in vivo.

- Phospholipid concentrations and diversity. As main ingredients to maintain the structure of vesicles [16], phospholipid concentrations need to be measured. Also, the category of phospholipid needs to be identified and contents of each phospholipid need measurement.

- Protein contents and diversity. Similar to phospholipids, the general protein contents should be measured. Furthermore, the diversity of proteins should also be studied, and this work can be done via proteomics analysis [10]. 
- Vesicle identification and stability. This issue is not very sensitive when natural EVs are assessed but should be taken special consideration when biomimetic EVs are used. External force may help cell membrane assembly into vesicles, but the efficiency is a problem. Some small cell membrane debris, other than vesicle, may exist. Also, after vesicles formed, the stability is a problem.

- Toxicity. The last concern about the clinical application of EVs is the possible immunotoxicity induced by EVs. In the previous studies, we found the EVs generated from nitrogen cavitation can activate HUVECs, while the function can be mitigated by the loaded anti-inflammatory drug. The following studies also demonstrate that the immunotoxicity may be caused by the exposed phosphatidylserine, originally locating on the inner surface of cell membrane, due to the external forces [16]. To avoid the impairment from immunotoxicity, antiinflammatory therapy may be necessary and helpful in some cases.

In the past decades, the research on EVs indicates that EVs are transforming the traditional nanoparticle-based drug delivery because EVs have the unique features. EVs have the excellent biocompatibility, long circulation time and low immunogenicity, and most importantly, they maintain their parent cell features that make them excellent drug carriers. Different to the conventional drug carrier, some EVs themselves perform as bioactive drug for disease therapy. Some special tissues, such as brain, can also be targeted by EVs, and no special targeting ligands are required. The above features confer EVs some outstanding advantages to be a drug carrier or disease treating agent. In summary, this review summarized the current most eminent challenges facing quality control towards commercialization of EVs. With advances in nanotechnology and immunology and genetic engineering, industrialization of EVs may become more and more realistic and eventually realize, and EVs will eventually turn to be a powerful tool for therapy of many human diseases.

\section{Acknowledgement}

None.

\section{Disclosure of Potential Conflicts of Interest}

No potential conflicts of interest were disclosed.

\section{References}

1. Thery C, KW Witwer, E Aikawa, MJ Alcaraz, JD Anderson, et al. (2018) Minimal information for studies of extracellular vesicles 2018 (MISEV2018): a position statement of the International Society for Extracellular Vesicles and update of the MISEV2014 guidelines. J Extracell Vesicles 7(1): 1535750.

2. Vader P, XO Breakefield, MJ Wood (2014) Extracellular vesicles: emerging targets for cancer therapy. Trends Mol Med 20(7): 385-393.

3. Raposo G, W Stoorvogel (2013) Extracellular vesicles: Exosomes, microvesicles, and friends. Journal of Cell Biology 200(4): 373-383.

4. Akers JC, D Gonda, R Kim, BS Carter, CC Chen (2013) Biogenesis of extracellular vesicles (EV): exosomes, microvesicles, retrovirus-like vesicles, and apoptotic bodies. Journal of Neuro-Oncology 113(1): 1-11.

5. Juan T, M Furthauer (2018) Biogenesis and function of ESCRT-dependent extracellular vesicles. Seminars in Cell \& Developmental Biology 74: 6677.

6. Brown L, JM Wolf, R Prados Rosales, A Casadevall (2015) Through the wall: extracellular vesicles in Gram-positive bacteria, mycobacteria and fungi. Nat Rev Microbiol 13: 620-630.

7. Ellis TN, MJ Kuehn (2010) Virulence and immunomodulatory roles of bacterial outer membrane vesicles. Microbiol Mol Biol Rev 74(1): 81-94.

8. Lee EY, DY Choi, DK Kim, JW Kim, JO Park, et al. (2009) Grampositive bacteria produce membrane vesicles: proteomics-based characterization of Staphylococcus aureus-derived membrane vesicles. Proteomics 9(24): 5425-5436.

9. Wang SH, J Gao, ZJ Wang (2019) Outer membrane vesicles for vaccination and targeted drug delivery. Wiley Interdisciplinary Reviews Nanomedicine and Nanobiotechnology 11(2).

10. Wang SH, J Gao, M Li, LG Wang, ZJ Wang (2018) A facile approach for development of a vaccine made of bacterial double-layered membrane vesicles (DMVs). Biomaterials 187: 28-38.

11. Gao J, DF Chu, ZJ Wang (2016) Cell membrane-formed nanovesicles for disease-targeted delivery. Journal of Controlled Release 224: 208-216.

12. Ingato D, JA Edson, M Zakharian, YJ Kwon (2018) Cancer Cell-Derived, Drug-Loaded Nanovesicles Induced by Sulfhydryl-Blocking for Effective and Safe Cancer Therapy. ACS Nano 12(9): 9568-9577.

13. Kanada M, MH Bachmann, JW Hardy, DO Frimannson, L Bronsart, et al. (2015) Differential fates of biomolecules delivered to target cells via extracellular vesicles. Proc Natl Acad Sci U S A 112(12): E1433-1442.

14. Qu JL, XJ Qu, MF Zhao, YE Teng, Y Zhang, et al. (2009) Gastric cancer exosomes promote tumour cell proliferation through PI3K/Akt and MAPK/ERK activation. Digestive and Liver Disease 41(12): 875-880.

15. Wang SH, XY Dong, J Gao, ZJ Wang (2018) Targeting Inflammatory Vasculature by Extracellular Vesicles. Aaps Journal 20(2): 37.

16. Gao J, SH Wang, ZJ Wang (2017) High yield, scalable and remotely drug-loaded neutrophil-derived extracellular vesicles (EVs) for antiinflammation therapy. Biomaterials 135: 62-73. 